\title{
Dosage des caséines du lait de vache par électrophorèse et par chromatographie liquide rapide d'échange d'ions (FPLC ${ }^{\circledR}$ ) : comparaison des résultats
}

\author{
JC Collin 1, A Kokelaar 1, O Rollet-Repecaud 1, \\ A Delacroix-Buchet 2
}

1 INRA, station de recherches en technologie et analyses laitières, BP 89, 39800 Poligny;

2 INRA, station de recherches laitières, 78350 Jouy-en-Josas, France

(Reçu le 28 septembre 1990; accepté le 25 février 1991)

\begin{abstract}
Résumé - L'analyse quantitative des diverses caséines du lait de vache a été réalisée par électrophorèse en gel de polyacrylamide-agarose $\mathrm{pH} 8,6$ et par chromatographie anionique à $\mathrm{pH} 8,0$. L'analyse de 118 échantillons individuels de lait prélevés dans un troupeau de 13 vaches durant une lactation a permis d'estimer les proportions de chaque caséine et de comparer les résultats des 2 méthodes. Une étude sur la capacité de fixation spécifique du colorant bleu de Coomassie R 250 sur chaque caséine a donné des coefficients moyens d'absorption par rapport à la caséine $\alpha \mathrm{s}_{1}$ prise comme référence $(1,00)$ de 0,98 pour la caséine $\alpha s_{2}$, de 0,95 pour la caséine $\beta$ et de 0,94 pour la caséine $\kappa$.

La comparaison des résultats entre les 2 méthodes d'analyse donne des coefficients de corrélation de 0,80 pour la fraction $\gamma_{1}$, de 0,77 pour la caséine $\beta$, de 0,74 pour la fraction $\gamma_{2}$ et de 0,65 pour les caséines $\alpha$ s. Les corrélations sont très faibles pour la caséine $\kappa$ et la fraction $\gamma_{3}$. L'étude des relations pouvant exister entre les diverses caséines du lait montre que les taux des 3 fractions $\gamma$ sont bien corrélés contrairement à d'autres résultats trouvés dans les fromages (Collin et al, 1987).
\end{abstract}

lait de vache / caséine / dosage / électrophorèse / chromatographie

Summary - Determination of caseins in cow's milk by electrophoresis and fast protein liquid chromatography (FPLC $\left.{ }^{8}\right)$ : comparison of results. Studies on quantitative determination of bovine milk caseins were performed using polyacrylamide-agarose gel electrophoresis ( $\mathrm{pH} 8.6)$ and anionic chromatography ( $\mathrm{pH}$ 8.0). One hundred and eighteen individual milks were sampled during a lactation period in a herd of 13 Montbeliard cows and analysed in order to estimate the amount of each casein and compare results obtained by each method. The uptake capacity of Coomassie blue $R 250$ dye by each casein was also studied using $\alpha s_{1}$ casein as standard. Differences between caseins were $<6 \%$.

Comparison of the 2 determination methods gave correlations between results of 0.80 for the $\gamma_{f}$ fraction, 0.77 for the $\beta$-casein, 0.74 for the $\gamma_{2}$ fraction, 0.65 for the $\alpha$ s-casein. Very weak correlations were found for the $\gamma_{3}$ fraction and the $\kappa$-casein. Study of the relationship between the different caseins showed good correlations in milk between the $3 \gamma$ fractions contrary to results found in cheeses (Collin et al, 1987). 


\section{INTRODUCTION}

La détermination de la teneur en protéines totales du lait est réalisée quotidiennement dans des laboratoires spécialisées à l'aide de méthodes parfaitement standardisés (Kjeldahl, colorimétrie ou spectrométrie dans l'infrarouge et l'ultraviolet).

Le lait est l'aliment dont les connaissances sur les protéines sont les plus avancées. En particulier, les séquences des amino-acides des 6 principales protéines du lait de vache ont été élucidées. Des revues scientifiques très détaillées sur les dosages des protéines du lait ont été publiées ces dernières années (Guillou et al, 1986; Ribadeau Dumas et Grappin, 1989).

Les protéines du lait se divisent en 2 grandes catégories, d'une part les caséines précipitables à $\mathrm{pH} 4,6$ formant la trame protéique du fromage et d'autre part, les protéines sériques solubles que l'on retrouve dans le lactosérum.

La séparation et la détermination des proportions des différentes caséines du lait a été le plus souvent réalisée à l'aide de techniques électrophorétiques (Haenlein et al, 1973; Randolph et al, 1974; Ng-Kwai-Hang et Kroeker, 1984; Collin et al, 1987). Le développement de la chromatographie liquide sous pression (HPLC, FPLC ${ }^{\circledR}$ ) a permis de séparer et de doser les principales caséines du lait en réduisant considérablement la durée d'une analyse (moins d'1 h). Ces séparations chromatographiques ont été le plus souvent réalisées sur colonne Mono Q (Pharmacia, Uppsala) (Andrews et al, 1985; Barrefors et al, 1985; Guillou et al, 1987). Au cours de ces travaux, la chromatographie n'a pas été employée à grande échelle comme méthode de routine. Les résultats publiés dans cet article représentent l'ana- lyse de 118 échantillons de laits individuels d'un troupeau de vaches montbéliardes par chromagraphie et électrophorèse, utilisées comme méthodes de routine, et la comparaison des résultats obtenus par ces 2 techniques d'analyse.

\section{MATÉRIEL ET MÉTHODES}

\section{Matériel}

Cent dix huit échantillons de lait ont été prélevés régulièrement pendant 1 année (11 prélèvements) sur un troupeau de 13 vaches montbéliardes. Moins de $3 \mathrm{~h}$ après la traite, ces échantillons ont été congelés et conservés à $-20^{\circ} \mathrm{C}$ jusqu'à leur traitement pour analyse.

Le système chromatographique FPLC (Pharmacia LCC 500) a été utilisé avec le passeur d'échantillon MV8 et une colonne échangeuse d'anions Mono Q HR 5/5 (Pharmacia).

Une cuve d'électrophorèse de $17 \times 13 \mathrm{~cm}$ (Sébia) équipée d'un système de refroidissement à $+10^{\circ} \mathrm{C}$ a servi à réaliser des migrations électrophorétiques horizontales.

\section{Méthodes}

\section{Préparation des échantillons}

Après décongélation, les échantillons de lait ont été écrémés par centrifugation à $10000 \mathrm{~g}$, pendant $10 \mathrm{~min}$. Le lait écrémé a été pesé puis acidifié à $\mathrm{pH} 4,6$ à l'aide d'une solution d'acide chlorhydrique $1 \mathrm{~N}$. Après quelques min d'agitation, les protéines précipitées ont été récupérées par centrifugation $(10000 \mathrm{~g}$, pendant 10 $\mathrm{min})$. Le culot humide a été pesé et, de manière à réaliser les analyses électrophorétiques et chromatographiques sur le même précipité sans décongélations successives, des quantités de $0,5 \mathrm{~g}$ de précipité ont été réparties dans des tubes et congelées à $-20^{\circ} \mathrm{C}$. 


\section{Analyses électrophorétiques}

Les électrophorèses ont été effectuées en gel d'acrylamide-bisacrylamide-agarose $(5 \%-0,5 \%$ $-0,8 \%)$ en tampon Tris/glycine $\left(0,075 \mathrm{~mol}^{-\mathrm{l}^{-1}}\right.$, 0,06 mol. $^{-1}, \mathrm{pH} 8,6$ ), urée $4,5 \mathrm{~mol}^{-\mathrm{I}^{-1}}$ et $0,2 \% \beta$ mercapto-éthanol. L'échantillon congelé de caséines précipitées $(0,5 \mathrm{~g})$ a été dissous dans 2,2 $\mathrm{ml}$ d'urée 9 mol. $\mathrm{l}^{-1}$ auxquels ont été ajoutés 40 $\mu \mathrm{l}$ de soude $3 \mathrm{~N}$ pour remonter le $\mathrm{pH}$ et $0,3 \%$ de $\beta$ mercapto-éthanol. La migration a duré 2 h 30 sous une tension de $25 \mathrm{~V} / \mathrm{cm}$.

Après coloration du gel au bleu de Coomassie R 250 puis déshydratation à $37^{\circ} \mathrm{C}$ sous feuilles de cellophane, l'analyse quantitative des plaques a été réalisée avec un densitomètre enregistreur (Vernon). La surface de chaque pic a été mesurée sous une longueur d'onde de 590 $\mathrm{nm}$ et exprimée en pourcentage de la surface totale de l'ensemble des pics (Collin et al, 1987).

\section{Analyses chromatographiques}

L'échantillon congelé de caséines précipitées $(0,5 \mathrm{~g})$ a été dissous dans $17 \mathrm{ml}$ de tampon Tris/ $\mathrm{HCl}\left(5,0.10^{-3} \mathrm{~mol}^{-I^{-1}} \mathrm{pH} 8,0\right)$ urée $\left(9 \mathrm{~mol}^{-1} \mathrm{I}^{-1}\right)$, azide de sodium $\left(1,5.10^{-3}\right.$ mol. $\left.1^{-1} \%\right)$ et dithiothréitol $\left(1,0.10^{-2} \mathrm{~mol} . \mathrm{I}^{-1}\right)$. L'échantillon a été ensuite ajusté à $\mathrm{pH} 8,0$, filtré sur un filtre de 0,22 $\mu \mathrm{m}$ et $500 \mu \mathrm{l}$ ont été déposés sur la colonne Mono Q Pharmacia. L'analyseur FPLC Pharmacia permet de réaliser les gradients d'élution chromagraphique à partir de 2 tampons $A$ et $B$. Ces tampons ont été préparés avec de l'eau UHQ traitée sur un système de filtration Elgostat (Appareil Elga). La composition du tampon A était : Tris/ $\mathrm{HCl} 5.10^{-3} \mathrm{~mol}^{-1} \mathrm{I}^{-1} \mathrm{pH} 8,0$ urée 4,5 mol. $1^{-1}$, azide de sodium $1,5.10^{-3}$ mol. $1^{-1}$ et dithiothréitol $6,4 \cdot 10^{-6} \mathrm{~mol}^{1} \mathrm{I}^{-1}$. Le tampon $\mathrm{B}$ conte-

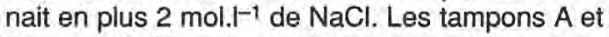
$B$ ont été dégazés et filtrés sous vide sur un filtre de 0,22 $\mu \mathrm{m}$ (Guillou et al, 1987). La détection des caséines a été effectuée à la sortie de la colonne à $280 \mathrm{~nm}$.

Après environ 20 séparations, la colonne a été lavée par le passage successif des solutions suivantes:

$-2 \mathrm{ml}$ d'acide acétique $75 \%$;

$-2 \mathrm{ml}$ de $\mathrm{NaCl} 2$ mol. $^{-1}$;

$-2 \mathrm{ml}$ de $\mathrm{NaOH} 2 \mathrm{~N}$;

$-2 \mathrm{ml}$ de $\mathrm{NaCl} 2 \mathrm{~mol}^{-1}{ }^{-1}$;

$-2 \mathrm{ml}$ de méthanol.
Les solutions ont été éluées avec de l'eau UHQ filtrée et dégazée. Pendant le lavage, le flux des solutions a été inversé. La colonne a ensuite été rééquilibrée avec une solution de $\mathrm{NaCl} 2 \mathrm{~mol}^{-1} \mathrm{I}^{-1}$ et 1 et à 2séparations de caséines ont généralement été nécessaires avant d'obtenir un profil chromatographique optimal.

\section{RÉSULTATS ET DISCUSSIONS}

\section{Analyses électrophorétiques}

\section{Fixation du colorant}

Afin de préciser la capacité de fixation du colorant par chaque caséine, nous avons déposé des quantités connues et croissantes de protéines purifiées sur un même gel (coloration et décoloration identiques). L'intensité de coloration estimée par un spectrophotomètre correspond à la surface du pic d'absorption. Nous avons répété les mesures sur 4 plaques différentes. Les coefficients moyens d'absorption du colorant pour chaque caséine exprimés par rapport à la caséine $\alpha s_{1}$ prise comme référence $(1,00)$ ont été respectivement de $0,98,0,95$ et 0,94 pour les caséines $\alpha s_{2}, \beta$ et $\kappa$. La différence maximale d'absorption entre les caséines $\alpha s_{1}$ et $\kappa$ n'a donc pas dépassé $6 \%$, alors que la répétabilité des mesures pour chaque caséine dépassait $10 \%$. Ces valeurs n'ont donc pas permis de conclure à des différences significatives d'absorption du colorant entre les diverses caséines et dans la suite de ce travail nous n'avons pas corrigé les résultats du spectrophotomètre.

\section{Résultats des séparations}

Les figures 1 et 2 montrent les résultats électrophorétiques obtenus avec des échantillons de lait et des échantillons de caséines purifiées. 


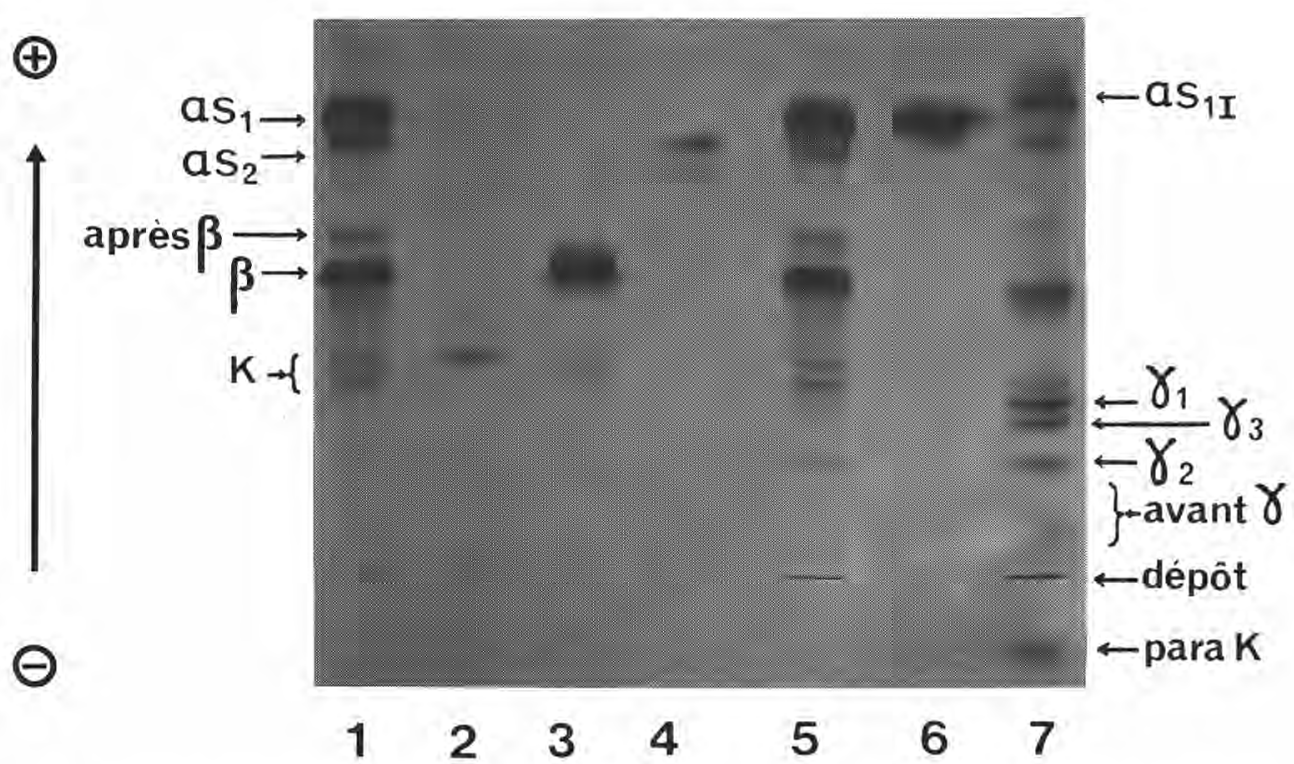

Fig 1. Analyse électrophorétique des caséinesdu lait de vache. 1: lait précipité à $\mathrm{pH} 4,6 ; 2$ : caséine $\kappa A$ purifiée; 3 : caséine $\beta$ purifiée; 4 : caséine $\alpha \mathrm{s}_{2}$ purifiée; 5 ; lait précipité à $\mathrm{pH} 4,6 ; 6$ : caséine $\alpha \mathrm{s}_{1}$ purifiée; 7: échantillon de fromage.

Polyacrylamide gel electrophoretic patterns of bovine milk. 1: milk precipitated at $\mathrm{pH} 4.6$; 2: (purified) $\kappa$ A-casein; 3: (purified) $\beta$-casein; 4: (purified) $\alpha \mathrm{s}_{2}$-casein; 5: milk precipitated at $\mathrm{pH} 4.6$; 6 : (purified) $\alpha s_{1}$-casein; 7: cheese sample.

Pour un échantillon de lait, nous identifions facilement 2 des 3 variants génétiques de la caséine $\kappa$ et les caséines $\beta, \alpha s_{2}$ et $\alpha s_{1}$. Dans le lait de 5 vaches sur 13 une bande de mobilité électrophorétique supérieure à la caséine $\beta$ a été enregistrée. Cette protéine migre comme la $\beta$ lactoglobuline, mais après 3 précipitations de l'échantillon à $\mathrm{pH} \mathrm{4,6}$ et lavages successifs, cette bande persiste et représente certaines fois jusqu'à $10 \%$ des protéines précipitables à $\mathrm{pH} 4,6$. Nous n'avons pas identifié cette protéine et dans la suite de cette étude nous l'avons regroupée avec la caséine $\beta$.
L'électrophorèse de caséines purifiées montre que les caséines $\kappa$ glycosylées semblent migrer sous forme de bandes peu colorées entre la caséine $\kappa$ non glycosylée et la caséine $\beta$. Le spectrophotomètre n'a pas la possibilité de différencier la caséine $\beta$ des bandes mineures situées avant cette caséine. Les lectures électrophorétiques peuvent ainsi sous-estimer les caséines $\kappa$ et surestimer légèrement les caséines $\beta$. L'analyse de la caséine $\alpha s_{2}$ purifiée montre 2 bandes que nous avons regroupées dans la suite de cette étude, pour déterminer la valeur de la caséine $\alpha s_{2}$. 


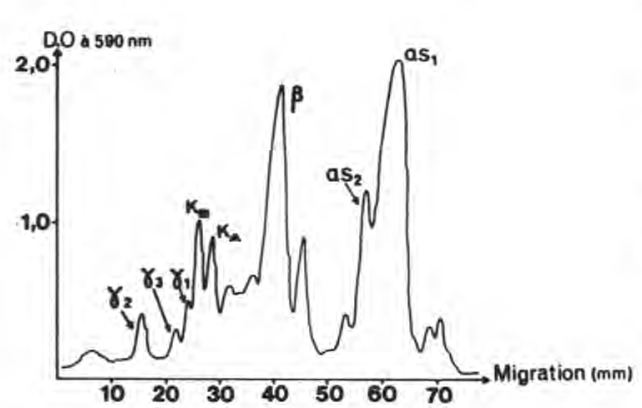

Fig 2. Séparation électrophorétique en gel d'acrylamide-bisacrylamide-agarose à $\mathrm{pH}$ 8,6 d'un échantillon de caséine entière obtenu par précipitation à $\mathrm{pH} 4,6$. Gel coloré au bleu de Coomassie R250. Lecture densitométrique des bandes d'électrophorèse à $590 \mathrm{~nm}$.

Electrophoretic separation in polyacrylamideagarose gel at $\mathrm{pH} 8.6$ of whole casein sample obtained by precipitation at $\mathrm{pH}$ 4.6. The gel was colored with Coomassie blue R250. Densitogram at $590 \mathrm{~nm}$.

\section{Répétabilité de la méthode électrophorétique}

La répétabilité de la méthode électrophorétique a été estimée à partir de l'analyse en double des 118 échantillons de lait. Le tableau I indique la moyenne de chaque caséine, l'écart type de répétabilité, la répétabilité (exprimée par l'écart maximal obtenu entre 2 doubles) et le coefficient de variation.

Pour les caséines principales $(\kappa, \beta$ et $\alpha s$ ), la répétabilité s'améliore par rapport aux premiers travaux de Collin et al (1987) avec un coefficient de variation égal ou inférieur à $5 \%$. Le regroupement de la bande $\beta$ avec la bande non identifiée de mobilité électrophorétique légèrement supérieure améliore la répétabilité des mesures réalisées individuellement sur chaque bande. Ce résultat tend à prouver que cette bande non identifiée pourrait être une partie de la caséine $\beta$. Une remarque similaire peut- être faite sur la répétabilité de la somme des $\alpha S$ par rapport à la répétabilité des mesures individuelles des caséines $\alpha S_{2}$ et $\alpha S_{1}$. En effet, la séparation parfois imparfaite de ces 2 caséines entraîne de mauvaises lectures au spectrophotomètre responsables d'une sous-estimation de la caséine $\alpha S_{2}$ et d'une surestimation de la caséine $\alpha S_{1}$ (fig 2).

\section{Analyses chromatographiques}

\section{Absorption à $280 \mathrm{~nm}$}

L'absorption à $280 \mathrm{~nm}$ n'est pas identique pour toutes les caséines. Les valeurs lues par l'intégrateur ont été corrigées en les multipliant par des facteurs déterminés par Guillou et al (1987) et valables pour le tampon utilisé. En prenant la caséine $\alpha s_{1}$ comme référence, ces facteurs pour les caséines $\alpha s_{2}, \beta$ et $\kappa$ sont respectivement de $1,02,2,14$ et 1,00 .

\section{Résultats des séparations}

La figure 3 schématise le profil chromatographique moyen des caséines lors de l'analyse d'un échantillon de lait. Le débit était de $2 \mathrm{ml} / \mathrm{min}$, le volume de l'échantillon déposé sur la colonne Mono Q (5/5) était de $500 \mu \mathrm{l}$ et la quantité totale des protéines déposées était de $15 \mathrm{mg}$. Le gradient d'élution retenu permet la séparation des principales caséines (Miranda, communication personnelle, 1989). L'élimination des protéines solubles par précipitation des caséines à $\mathrm{pH}$ 4,6 permet de mettre en évidence les fractions $\gamma\left(\gamma_{1}, \gamma_{2}\right.$ et $\left.\gamma_{3}\right)$.

Les pics correspondant aux fractions $\gamma_{2}$ et $\gamma_{3}$ sortent les premiers de la colonne Mono $Q$ et sont en général uniques et très symétriques. Par contre, le pic de la frac- 
Tableau I. Répétabilité de l'analyse électrophorétique des caséines du lait de vache. (av $\gamma=$ avant $\gamma$; $\beta T=\beta$ Total; ap $\beta=$ après $\beta$.

Repeatability of the electrophoretic analysis of bovine milk caseins.

$\begin{array}{lllllllllllll}\text { Caséines } & a v \gamma & \gamma_{2} & \gamma_{3} & \gamma_{1} & \kappa & \beta T & \text { ap } \beta & \beta+a p \beta & \alpha s_{2} & \alpha s_{1} & \Sigma \alpha s\end{array}$

$\begin{array}{lllllllllllll}\begin{array}{l}\text { Valeur moyenne } \\ \text { en \% de la surface } \\ \text { total des pics }(\bar{x})\end{array} & 1,04 & 2,00 & 1,29 & 1,23 & 9,28 & 31,37 & 6,19 & 37,56 & 10,10 & 37,50 & 47,60 \\ & & & & & & & & & & & \\ \begin{array}{l}\text { Répétabilité } \\ \text { Écart type }(\sigma r)\end{array} & 0,48 & 0,34 & 0,30 & 0,13 & 0,49 & 1,32 & 0,71 & 1,41 & 1,03 & 1,65 & 1,61 \\ r=-2,83 \cdot \sigma r & 1,36 & 0,97 & 0,85 & 0,38 & 1,39 & 3,73 & 2,00 & 3,98 & 2,92 & 4,67 & 4,54 \\ \text { CV }=\frac{\sigma r}{\bar{x}} \times 100 & 46,10 & 17,00 & 23,24 & 10,80 & 5,30 & 4,20 & 11,50 & 3,75 & 10,20 & 4,40 & 3,37\end{array}$

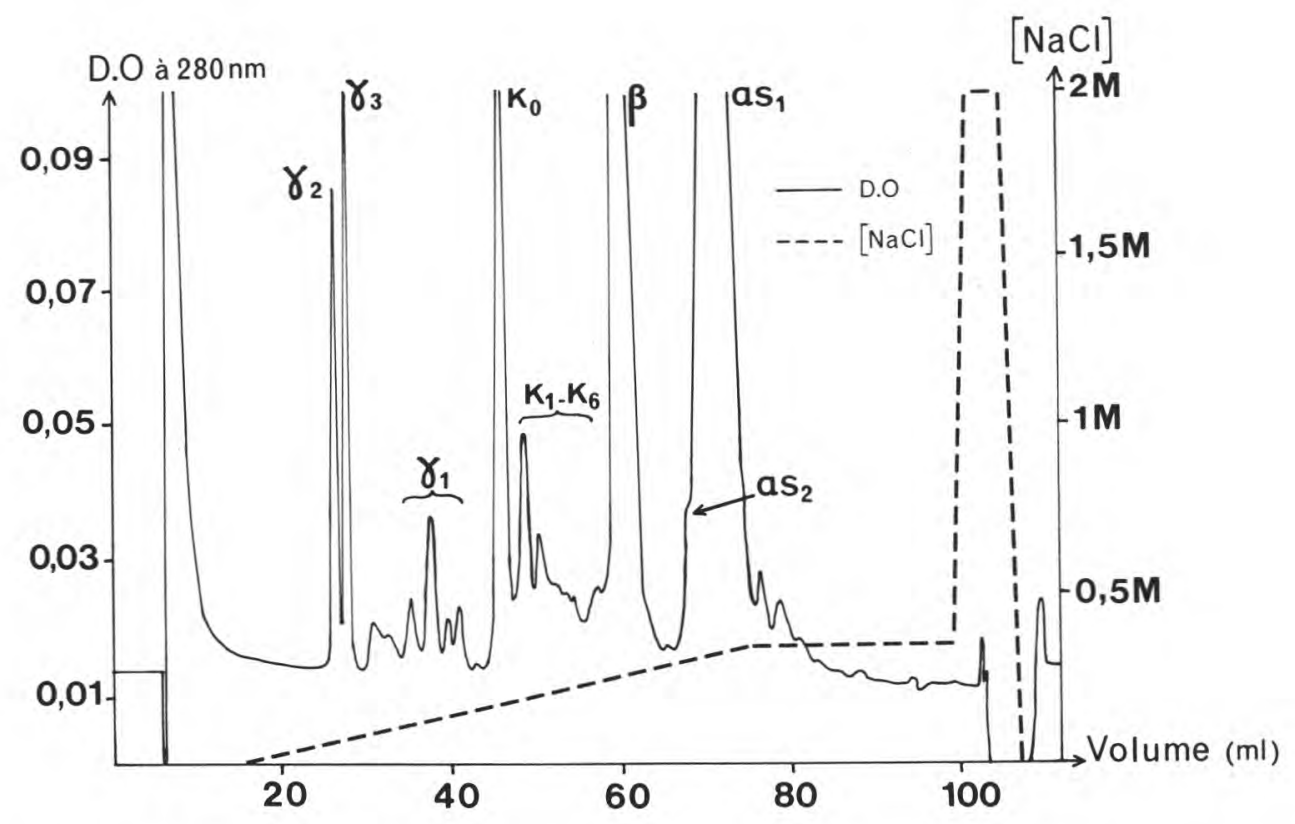

Fig 3. Séparation chromatographique par FPLC sur colonne mono Q d'un échantillon de caséine entière bovine. Débit $2 \mathrm{ml} / \mathrm{min}$, tampon Tris-HCl $510^{-3} \mathrm{~mol}^{-1} \mathrm{I}^{-1}$, urée $4,5 \mathrm{~mol} . \mathrm{I}^{-1}$, dithiothréitol $6,410^{-5}$

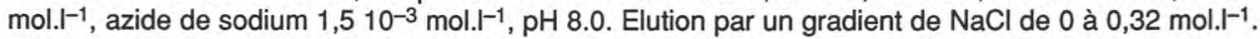
Echantillon dissous dans le tampon avec urée 9 mol..$^{-1}$ et dithiothréitol $10^{-2} \mathrm{~mol}^{-1} \mathrm{I}^{-1}$.

FPLC chromatography of whole bovine casein sample carried out on a Mono $Q$ column using $510^{-3}$ mol..$^{-1}$ Tris- $\mathrm{HCl}$ buffer, $\mathrm{pH} 8.0$, containing 4.5 mol..$^{-1}$ urea, $6.410^{-5} \mathrm{~mol}^{-\mathrm{H}^{-1}}$ dithiothreitol, $1.510^{-3}$ mol..$^{-1} \mathrm{NaN3}$ at a flow rate of $2 \mathrm{ml} / \mathrm{min}$ with a linear $\mathrm{NaCl}$ gradient (0-0.32 mol. $\left.\mathrm{H}^{-1}\right)$. Sample was dissolved in 9 mol..$^{-1}$ urea buffer containing $10^{-2}$ mol..$^{-1}$ dithiothreitol. 
tion $\gamma_{1}$ est toujours entouré de petits pics que nous avons regroupés avec le pic principal $\gamma_{1}$ pour donner la valeur des fractions $\gamma_{1}$ en chromatographie FPLC.

L'enregistrement d'un chevauchement des 2 pics de caséine ko (non glycosylée) avec les premiers pics de caséine $\kappa$ glycosylée, lors de l'analyse d'un échantillon de lait d'une vache hétérozygote pour la caséine $\kappa$ nous a obligés à regrouper tous les pics de caséines $\kappa$ au cours de cette étude. D'autres travaux (Dalgleish, 1986) mettent également en évidence, sur colonne anionique, les variants $A$ et $B$ de la caséine $\kappa$. Cette technique analytique permet ainsi de différencier les variants des différents animaux.

Avec les laits du troupeau retenu dans cette étude, nous n'avons pas pu séparer la caséine $\alpha s_{2}$ de la caséine $\alpha s_{1}$. Seul un épaulement au début du pic $\alpha s_{1}$ a été enregistré pour certains échantillons. Des essais de séparation chromatographique à $40{ }^{\circ} \mathrm{C}$ n'ont pas permis d'enregistrer les améliorations souhaitées.

\section{Répétabilité de la méthode de séparation chromatographique}

Comme pour l'analyse électrophorétique, les 118 échantillons de lait ont été analy- sés en double par la méthode chromatographique. Le tableau II indique la moyenne de chaque caséine, l'écart type de répétabilité, la répétabilité et le coefficient de variation.

Les coefficients de variation des 3 caséines principales, $\kappa, \beta$ et $\alpha \mathrm{S}$, sont respectivement de $6,67,2,58$ et 2,92 . Deux analyses effectuées l'une après l'autre, donnent en général des résultats toujours très répétables, tandis que le même échantillon analysé en double à plusieurs jours d'intervalle peut donner des résultats peu reproductibles. En effet, le lavage et le rééquilibrage de la colonne sont des opérations importantes mais relativement longues qu'il s'avère impossible de réaliser tous les 4 ou 5 échantillons. Lors de ce travail, la colonne était lavée environ tous les 20 échantillons et les échantillons ont tous été analysés, en double, sur 2 j différents avec un ordre de passage déterminé au hasard.

\section{Comparaison des deux méthodes}

La comparaison des résultats obtenus par les méthodes chromatographique et électrophorétique a été faite en calculant le coefficient de corrélation existant entre les

Tableau II. Répétabilité de l'analyse chromatographique des caséines du lait de vache.

Repeatability of the chromatographic analysis of bovine milk caseins.

\begin{tabular}{|c|c|c|c|c|c|c|}
\hline Caséines & $\gamma_{2}$ & $\gamma_{3}$ & $\gamma_{1}$ & $\Sigma$ & $\beta$ & $\Sigma \alpha s$ \\
\hline $\begin{array}{l}\text { Valeur moyenne } \\
\text { en } \% \text { de la surface } \\
\text { totale des pics }(\bar{x})\end{array}$ & 1,30 & 2,35 & 1,78 & 10,63 & 38,70 & 45,20 \\
\hline Répétabilité & & & & & & \\
\hline Écart type (бr) & 0,23 & 0,53 & 0,61 & 0,71 & 1,00 & 1,32 \\
\hline $\begin{array}{c}r=2,83 \cdot \sigma r \\
\sigma r\end{array}$ & 0,66 & 1,49 & 1,73 & 2,01 & 2,82 & 3,73 \\
\hline $\mathrm{CV}=\frac{-}{\bar{x}} \times 100$ & 18,00 & 22,40 & 34,30 & 6,67 & 2,58 & 2,92 \\
\hline
\end{tabular}


2 séries de résultats pour les principales caséines (fractions $\gamma_{1}, \gamma_{2}, \gamma_{3}$ et caséines $\kappa$, $\beta$ et $\alpha$ ) (tableau III). Ces résultats doivent être analysés avec une certaine prudence en raison de la faible amplitude de variation des taux des diverses caséines entre échantillons de lait.

Les courbes $4 \mathrm{~A}, 4 \mathrm{~B}$ et $4 \mathrm{C}$ montrent les relations entre les valeurs des fractions $\gamma$ analysées par les 2 méthodes. Le très faible coefficient de corrélation $(0,237)$ obtenu pour la fraction $\gamma_{3}$ peut-être expliqué par l'existence d'échantillons dont les résultats divergent particulièrement en fonction de la méthode utilisée. Les moyennes des valeurs de la fraction $\gamma_{3}$ sont d'ailleurs celles qui diffèrent le plus $(1,29$ et 2,35$)$ par rapport aux moyennes des autres fractions $\gamma$. La mauvaise répétabilité de la mesure de la fraction $\gamma_{1}$ en chromatographie s'explique par la multitude de pics sommés. Par contre, le coefficient de corrélation entre les valeurs des 2 méthodes d'analyse pour la fraction $\gamma_{1}$ est le meilleur de toutes les caséines mesurées $(0,797)$.

L'analyse des caséines $\kappa$ pose une interrogation majeure sur la signification des résultats, au regard du très faible coefficient de corrélation $(0,042)$ mesuré entre les 2 méthodes d'analyse. Les valeurs des échantillons individuels trouvées par les 2 méthodes (fig 5) sont différentes les unes des autres. Les 2 moyennes générales semblent quant à elles concorder, l'écart observé pouvant être dû par ailleurs au fait que la méthode électrophorétique sousestime les caséines $\kappa$ en comptabilisant une partie des caséines $\kappa$ glycosylées avec les caséines $\beta$. De plus, il est bien connu que les glycoprotéines fixent mal le colorant bleu de Coomassie R 250 (fig 1). Les valeurs des échantillons varient de 7,16 à $15,98 \%$, alors qu'en électrophorèse ces mêmes échantillons sont situés entre 5,98 et $12,94 \%$. Le coefficient de variation de répétabilité légèrement plus élevé en chromatographie qu'en électrophorèse $(6,67 \%$ au lieu de $5,30 \%)$ peut s'expliquer par le fait qu'en chromatographie nous sommons plusieurs petits pics de caséines $\kappa$ glycosylées ce qui est un risque d'erreur plus important que la lecture d'une simple bande colorée lors de l'électrophorèse.

En électrophorèse, si nous regroupons avec la bande principale de caséines $\beta$, la bande située juste après celle-ci, les moyennes de la caséine $\beta$ obtenues par les 2 méthodes d'analyse deviennent

Tableau III. Comparaison des résultats des analyses électrophorétique et chromatographique des caséines du lait de vache.

Comparison of electrophoresis and chromatography of caseins from bovine milks.

\begin{tabular}{|c|c|c|c|c|c|c|c|}
\hline & & $\gamma_{2}$ & $\gamma_{3}$ & $\gamma_{1}$ & $\kappa$ & $\beta$ & $\Sigma \alpha s$ \\
\hline \multirow{2}{*}{$\begin{array}{l}\text { Valeur } \\
\text { moyenne } \\
\text { relative }\end{array}$} & électrophorèse & 2,00 & 1,29 & 1,23 & 9,28 & 37,56 & 47,60 \\
\hline & FPLC & 1,30 & 2,35 & 1,78 & 10,63 & 38,70 & 45,20 \\
\hline $\begin{array}{l}\text { Coefficient de } \\
\text { variation de }\end{array}$ & électrophorèse & 17,00 & 23,20 & 10,80 & 5,30 & 3,75 & 3,37 \\
\hline répétabilité (\%) & FPLC & 18,00 & 22,40 & 34,30 & 6,67 & 2,58 & 2,92 \\
\hline \multicolumn{2}{|c|}{$\begin{array}{l}\text { Coefficient de corrélation } \\
\text { entre les } 2 \text { méthodes }\end{array}$} & 0,739 & 0,237 & 0,797 & 0,042 & 0,772 & 0,650 \\
\hline
\end{tabular}



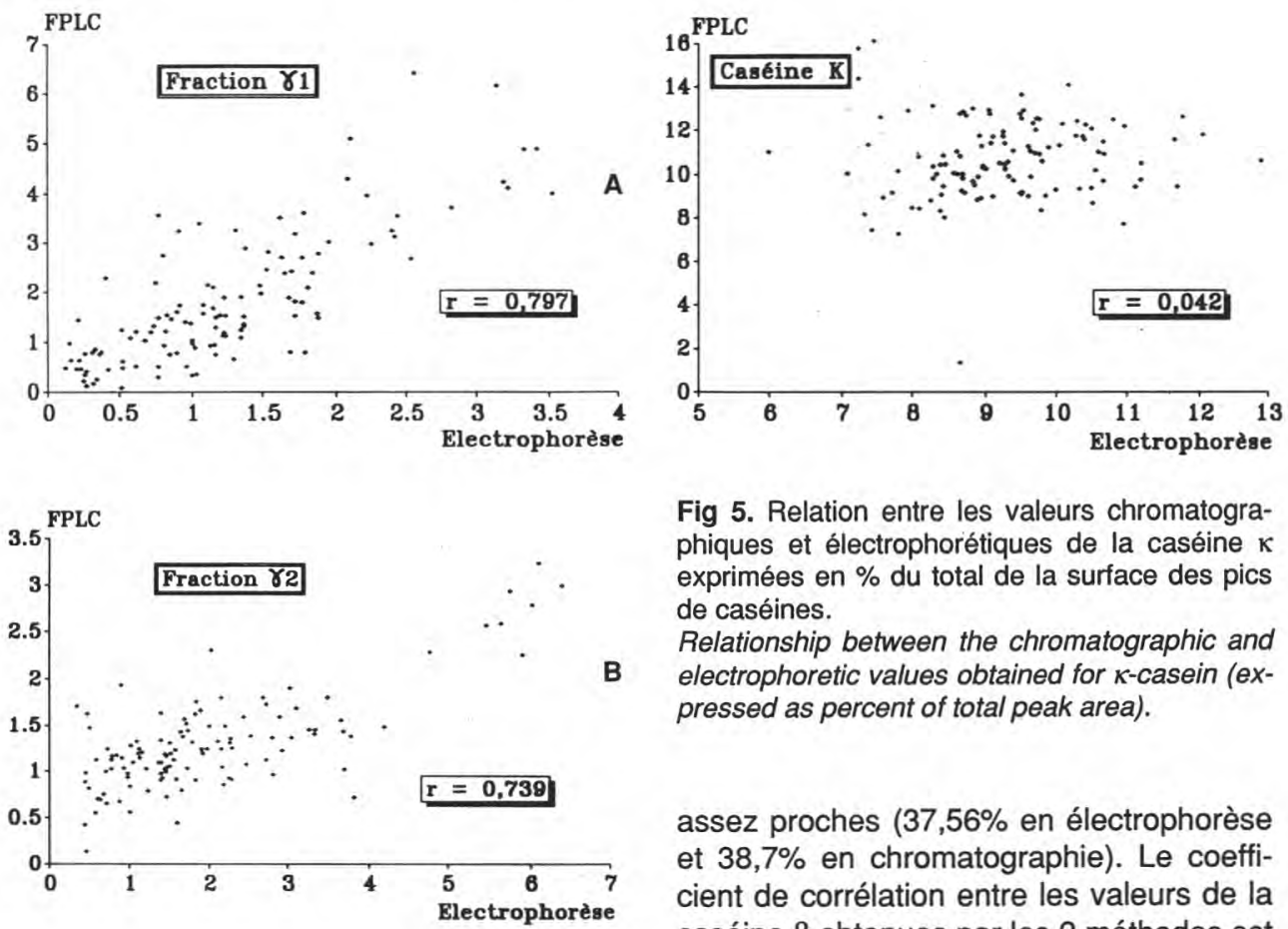

Fig 5. Relation entre les valeurs chromatographiques et électrophorétiques de la caséine $\kappa$ exprimées en \% du total de la surface des pics de caséines.

Relationship between the chromatographic and electrophoretic values obtained for $k$-casein (expressed as percent of total peak area).

assez proches $(37,56 \%$ en électrophorèse et $38,7 \%$ en chromatographie). Le coefficient de corrélation entre les valeurs de la caséine $\beta$ obtenues par les 2 méthodes est de 0,772 (fig 6A).

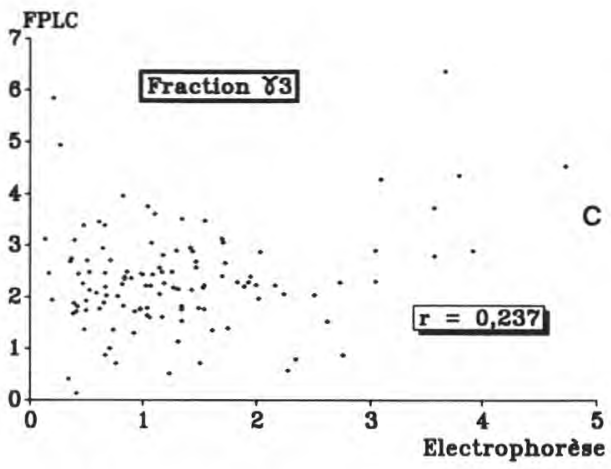

Fig 4. Relation entre les valeurs chromatographiques et électrophorétiques des fractions $\gamma$ exprimées en \% du total de la surface des pics de caséines. A: fraction $\gamma_{1}$; B: fraction $\gamma_{2} ; C$ : fraction $\gamma_{3}$.

Relationship between the chromatographic and electrophoretic values obtained for the $\gamma$ fractions (expressed as percent of total peaks area). $A: \gamma_{1}$ fraction; $B: \gamma_{2}$ fraction; $C: \gamma_{3}$ fraction.

Pour les caséines $\alpha$, la comparaison des résultats moyens trouvés par les 2 méthodes n'est pas aussi satisfaisante $(47,6 \%$ en électrophorèse et $45,2 \%$ en chromagraphie) et le coefficient de corrélation est inférieur $(0,65)$ à celui mesuré entre les valeurs de la caséine $\beta$ (fig $6 \mathrm{~B}$ ).

La comparaison statistique des moyennes obtenues avec les 2 méthodes de mesure pour les différentes caséines, à l'aide du test de Student, montre que dans tous les cas les moyennes sont significativement différentes au seuil de $1 \%$.

\section{Relations entre les variations des diverses caséines}

Le tableau IV indique les coefficients de corrélation existant entre les valeurs élec- 

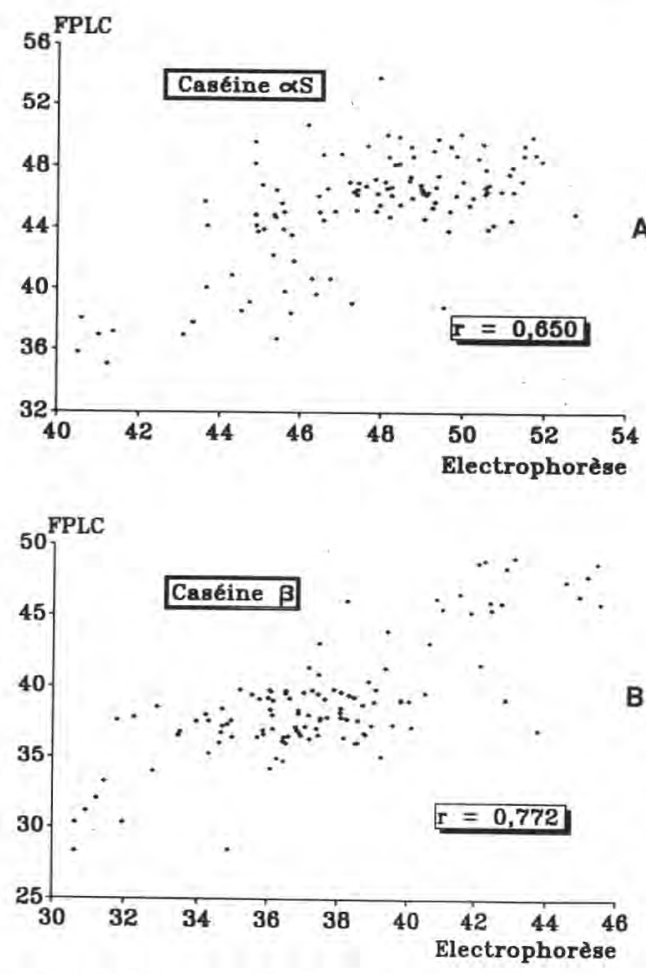

Fig 6. Relation entre les valeurs chromatographiques et électrophorétiques des caséines $\beta$ et $\alpha$ exprimées en $\%$ du total de la surface des pics de caséines. A: caséine $\beta$; B: caséine $\alpha$ s. Relationship between the chromatographic and electrophoretic values obtained for $\beta$ - and $\alpha$ scaseins (expressed as percent of total peak area). A: $\beta$-casein; $B$ : $\alpha$ s-casein.

trophorétiques des différentes caséines. Nous constatons que la meilleure corrélation est celle qui existe entre les teneurs des fractions $\gamma_{2}$ et $\gamma_{3}(0,943)$. Ce résultat confirme le travail publié par Collin et al (1987) et laisse à penser que ces 2 caséines sont produites par des systèmes enzymatiques semblables ou très proches. Nous notons également de bonnes corrélations entre la fraction $\gamma_{1}$ et respectivement les fractions $\gamma_{2}(0,910), \gamma_{3}(0,835)$ et la caséine $\beta(0,788)$. Dans le fromage de
Comté en fin d'affinage, la fraction $\gamma_{1}$ apparaissait indépendante des fractions $\gamma_{2}, \gamma_{3}$ et de la caséine $\beta$ (Collin et al, 1987). Cette constatation semble montrer que l'hydrolyse de la caséine $\beta$, pour donner naisA sance aux fractions $\gamma_{1}, \gamma_{2}$ et $\gamma_{3}$ dans le lait, est régie par des systèmes enzymatiques identiques ou très voisins, mais qu'ensuite l'apparition de la fraction $\gamma_{1}$ dans le fromage s'amplifie sous l'action de systèmes enzymatiques indépendants des premiers. L'hydrolyse de la caséine $\beta$ dans le lait est attribuée à l'activité de la plasmine, mais dans les fromages à pâte cuite pressée, le système enzymatique qui hydrolyse la caséine $\beta$ pour donner la fraction $\gamma_{1}$ est encore inconnu. Lors de l'analyse des fromages, les valeurs élevées des fractions $\gamma_{1}$ pourraient aussi s'expliquer par un artefact dû à une fraction inconnue de mobilité électrophorétique identique à celle de la fraction $\gamma_{1}$. Les corrélations enregistrées entre les valeurs des caséines trouvées par l'analyse chromatographique ont aussi été calculées, mais les coefficients de corrélation sont tous apparus inférieurs à ceux trouvés avec les résultats des analyses électrophorétiques.

\section{CONCLUSION}

La séparation et le dosage des caséines du lait de vache ont été réalisés par 2 méthodes différentes : l'électrophorèse et la chromatographie sur résine anionique (Appareil FPLC ${ }^{\circledR}$ Pharmacia).

Le pourcentage moyen annuel de chaque classe de caséines du lait de vache a pu être déterminé à partir d'échantillons prélevés mensuellement pendant un an sur 13 vaches. La somme des fractions $\gamma$ est apparue élevée par rapport aux valeurs annoncées dans la littérature $(4,52 \%$ en électrophorèse et $5,43 \%$ en chromatographie). La méthode électrophorétique 
Tableau IV. Coefficients de corrélation existant entre les valeurs des caséines du lait de vache estimées par électrophorèse.

Correlations between the amounts of the different milk caseins estimated by the electrophoretic method.

\begin{tabular}{lrrrrrrr}
\hline & \multicolumn{1}{c}{$\gamma_{2}$} & \multicolumn{1}{c}{$\gamma_{3}$} & $\gamma_{1}$ & $\kappa$ & $\beta$ & $\Sigma \alpha s$ \\
\hline av $\gamma$ & 1,000 & & & & & & \\
$\gamma_{2}$ & 0,538 & 1,000 & & & & & \\
$\gamma_{3}$ & 0,573 & 0,943 & 1,000 & & & & \\
$\gamma_{1}$ & 0,352 & 0,910 & 0,835 & 1,000 & & & \\
$\kappa$ & $-0,400$ & $-0,372$ & $-0,400$ & $-0,367$ & 1,000 & & \\
$\beta$ & $-0,261$ & $-0,684$ & $-0,597$ & $-0,788$ & 0,229 & 1,000 & \\
$\Sigma \alpha$ & $-0,384$ & $-0,255$ & $-0,334$ & $-0,022$ & $-0,147$ & $-0,456$ & 1,000 \\
\hline
\end{tabular}

semble sous-estimer la caséine $\kappa$ par rapport à la méthode chromatographique $(9,28 \%$ en électrophorèse et $10,63 \%$ en chromagraphie).

En électrophorèse, les diverses caséines absorbent des quantités de colorant bleu de Coomassie R 250 assez voisines. Les différences maximales d'absorption entre les 4 principales caséines n'excèdent pas $6 \%$.

La méthode chromatographique ne permet pas de doser en routine les caséines $\alpha s_{1}$ et $\alpha s_{2}$ à cause de la mauvaise résolution de ces 2 pics.

La comparaison des résultats obtenus par les 2 méthodes de dosage donne le meilleur coefficient de corrélation pour la fraction $\gamma_{1}$ et le plus mauvais pour la caséine $\kappa$.

L'estimation des proportions des différentes caséines du lait se réalise par des méthodes qui restent encore imprécises, et si aujourd'hui l'électrophorèse permet de tirer le maximum de renseignements, la chromatographie devrait, grâce à ses possibilités d'évolution, apporter à l'avenir les améliorations nécessaires à ces analyses.

\section{REMERCIEMENTS}

Nous tenons à exprimer nos remerciements aux personnes de la station de recherches laitières de Jouy-en-Josas qui nous ont fourni les échantillons de caséines purifiées et les conseils pour les séparations chromatographiques.

\section{RÉFÉRENCES}

Andrews AT, Taylor MD, Owen AJ (1985) Rapid analysis of bovine milk proteins by fast protein liquid chromatography. $J$ Chromatogr $348,177-185$

Barrefors $P$, Ekstrand B, Fagerstam L, LarssonRaznikiewicz M, Schaar J, Steffner P (1985) Fast protein liquid chromatography (FPLC) of bovine caseins. Milchwissenchaft 40, 257260

Collin JC, Berdagué JL, Dognin-Bergeret $M$, Grappin R (1987) Affinage et qualité du gruyère de Comté. IV. Étude de la protéolyse. Lait 67, 299-318

Dalgleish DG (1986) Analysis by fast protein Iiquid chromatography of variants of $\mathrm{k}$-casein and their relevance to micellar structure and renneting. J Dairy Res 53, 43-51

Guillou H, Pélissier JP, Grappin R (1986) Méthodes de dosage des protéines du lait de vache. Lait 66, 143-175 
Guillou H, Miranda G, Pélissier JP (1987) Analyse quantitative des caséines dans le lait de vache par chromatographie liquide rapide d'échange d'ions (FPLC). Lait 67, 135-148

Haenlein GFW, Schultz LH, Zikakis JP (1973) Composition of proteins in milk with varying leucocyte contents. J Dairy Sci 56, 10171024

Ng-Kwai-Hang KF, Kroeker EM (1984) Rapid separation and quantification of major ca- seins and whey proteins of bovine milk by polyacrylamide gel electrophoresis. J Dairy Sci 67, 3052-3056

Randolph HE, Erwin RE, Richter RL (1974) Influence of mastitis on properties of milk. VII. Distribution of milk proteins. J Dairy Sci 57, 15-18

Ribadeau Dumas B, Grappin R (1989) Milk protein analysis. Lait $69,357-416$ 